\title{
A Survey Study on Rheumatologist Consultation Time in Korean Hospitals
}

\author{
Hyun Ah Kim, M.D., Ph.D., ${ }^{1,2}$, Min Gwan Kim ${ }^{3}$ \\ ${ }^{1}$ Division of Rheumatology, Department of Internal Medicine, Hallym University Sacred Heart Hospital, Anyang, \\ ${ }^{2}$ Institute for Skeletal Aging, Hallym University, ${ }^{3}$ College of Medicine, Hallym University, Chuncheon, Korea
}

\begin{abstract}
Objective. In Korea, short physician consultation time has been a cause of concern as it compromises the care provided. This study was aimed at finding the actual and optimal consultation time per patient for rheumatology outpatient clinic in Korea. Methods. The surveys were prepared based on a study of the literature and consisted of multiple-choice questions as well as additional open questions. Surveys were conducted from November to February, 2018 2019. Rheumatologist members of the Korean College of Rheumatology were invited to complete the web-based survey as well as paper survey. Results. The mean duration of consultation allocated to a new and an established patient was found to be 12.3 and 4.8 minutes, respectively, which corresponded to only $22 \% \sim 35.3 \%$ of perceived optimal consultation time. On the other hand, the intrusion of physician autonomy for optimal patient care by the hospital executive was manifest such that only $4.7 \%$ responded discretion in allocating consultation time for patients and that $61.3 \%$ replied that they have been restrained from keeping the adequate outpatient clinic volumes. Sixty six percent of respondents replied that insufficient consultation time affects patient safety including errors in prescription. Conclusion. Rheumatology consultation time is very insufficient compared to optimal situation. A drastic change in health care policy promoting good quality of care, such as appropriate compensation which guarantees sufficient consultation time, as well as strong policy to control excessive profit-driven management policy of the hospitals is urgently needed. (J Rheum Dis 2020;27:45-50)
\end{abstract}

Key Words. Quality of health care, Rheumatology, Ambulatory care

\section{INTRODUCTION}

Physician consultation time is a critical factor influencing patient and doctor relationship and the quality of care that follows. It is a quality indicator used by the World Health Organization [1], and several countries following the International Network for the Rational Use of Drugs guideline set the consultation length as a quality standard [1]. A systematic literature review showed that increased consultation length is associated with more accurate diagnosis of psychological problems [2]. A phase 2 exploratory cluster randomized controlled trial with eight general practices in Glasgow showed that longer consultation is a cost-effective way to protect quality of life for multimorbid patients in deprived areas [3]. On the other hand, a Cochran review of 7 articles describing five UK trials showed that short term changes in the primary care consultation time did not lead to consistent differences in problem recognition, examination, prescribing, referral, investigation rates or patient satisfaction [4]. The relationship between the quality of care and physician consultation time would depend on a variety of factors, such as the patient profile, the specialty and the absolute length of consultation time. The majority of patients taken care of by a rheumatologist have arthritis, the leading cause of disability globally. For example, osteoarthritis is the most common form of arthritis affecting the elderly, and was ranked as the 11th leading cause of

Received : August 16, 2019, Revised : October 13, 2019, Accepted : October 15, 2019

Corresponding to : Hyun Ah Kim (1Dhttp://orcid.org/0000-0002-9318-7446

Division of Rheumatology, Department of Internal Medicine, Hallym University Sacred Heart Hospital, 22 Gwanpyeong-ro 170beon-gil, Dongan-gu, Anyang 14068, Korea. E-mail : kimha@hallym.ac.kr

Copyright (c) 2020 by The Korean College of Rheumatology. All rights reserved.

This is an Open Access article, which permits unrestricted non-commerical use, distribution, and reproduction in any medium, provided the original work is properly cited. 
years lived with disability (YLD) globally, with higher rank (6th) especially among Asian countries [5]. In addition, autoimmune diseases, such as systemic lupus erythematosus (SLE) are not only complex conditions with diverse constellation of symptoms and signs, but also the leading cause of chronic pain, which causes multidimensional problem including psychological and social changes. Considering the multi-morbidity inherent in old age of patients taken care of by rheumatologists into account, it seems obvious that rheumatologic care warrants a sufficient consultation time.

There is a paucity of data on what constitutes optimal consultation time for rheumatologic care, however. In a previous report covering $28,570,712$ primary care consultations in 67 countries, it was found out that 18 countries representing about $50 \%$ of the global population spent 5 minutes or less with their primary care physicians, which raised significant concern since such a short consultation length is likely to adversely affect patient healthcare, physician workload and stress [1]. In Korea, however, consultation time not only for primary care physician, but also for tertiary care is characterized by its shortness and as a result, the unfortunate derision " 3 -minuteconsultation" has been widespread for a long time.

This study was aimed at finding the actual consultation time per patient for rheumatology outpatient clinic in Korea. The consultation length deemed optimal for $2 \mathrm{ex}-$ emplar rheumatologic disease, rheumatoid arthritis (RA) and SLE, was subsequently assessed by a survey of rheumatologists working in hospitals.

\section{MATERIALS AND METHODS}

\section{Survey design and data collection}

The surveys were prepared based on a study of the literature and consisted of multiple-choice questions as well as additional open questions. For some questions of the survey, participants could indicate more than one answer, while for others, only a single answer only was accepted. The surveys comprised 32 questions in five sections (Supplementary Figure 1). The first section addressed physician characteristics (age, years since rheumatology specialist certification, and practice site). The second section queried practice characteristics, such as the number of out-patient clinic sessions per week, and the number of patients (total and new) per each clinic sessions, and the third section queried actual consultation time allocated for each patient (new and established, separately). The fourth section enquired perceived appropriate consultation time for RA and SLE, and the fifth, problems with patient care arising from current actual consultation time including compromise in quality of care and overuse of laboratory tests. Additionally, rheumatologists were asked about discretion in allocation of consultation time, delegation of clinical activity to other health care personnel and psychological and physical experience during outpatient clinics. Informed consent was not obtained and survey data were collected anonymously both electronically and in paper. Pilot paper survey in 16 rheumatologist participants, were conducted. Participants filled in the survey and wrote their remarks in a survey review report. The pilot studies showed good comprehensibility of the questions and technical functioning of the surveys. The web surveys were created using Google Forms (https:// docs.google.com/forms/).

\section{Inclusion of rheumatologists}

Surveys were conducted from November to February, 2018 2019. Rheumatologist members of the Korean College of Rheumatology (KCR) were invited by email to complete the web-based survey (estimated 219 personal email invitations). The invitations contained a link to the web survey. For the perceived difference in practice pattern, only members who worked in hospitals were included. In addition, paper survey was conducted at the annual meeting of KCR on the 16th, November 2018. Duplicate response was discouraged.

Table 1. Characteristics of the survey respondents and practice pattern $(n=106)$

\begin{tabular}{lc}
\hline \hline \multicolumn{1}{c}{ Variable } & Value \\
\hline Age $(\mathrm{yr})$ & $45.6 \pm 7.2$ \\
Years of rheumatology practice $(\mathrm{yr})$ & $11(1 \sim 38)$ \\
Number of outpatient clinic session/week & $5.1 \pm 1.5$ \\
Hours of outpatient clinic session/week & $22(3 \sim 46)$ \\
Number of patients per outpatient clinic & $40(10 \sim 200)$ \\
$\quad$ session & \\
Number of new patients per outpatient & $5(2 \sim 12.5)$ \\
$\quad$ clinic session & $12.3 \pm 5.4$ \\
Time allocated to a new patient (min) & $4.8 \pm 1.8$ \\
Time allocated to an established & \\
$\quad$ patient (min) & $68(64.1)$ \\
Tertiary hospital &
\end{tabular}

Values are presented as mean \pm standard deviation, median (range) or number (\%), unless specified otherwise. 


\section{Statistical analyses}

Descriptive statistics were used to quantify response frequencies and means or medians. Unless otherwise specified, frequencies are reported relative to the total number of respondents completing each question. Analysis was performed using Microsoft Excel Version 14.6.7 (Microsoft Corp., Redmond, WA, USA).

\section{RESULTS}

A total of 106 rheumatologists responded (78 online, 28 paper, response rate $48.4 \%$ ). Table 1 shows the physician and practice characteristics of the respondents. Average age of the respondents was 45.6 years (standard deviation [SD], 7.2) and median duration of rheumatology practice was 11 years (range $1 \sim 38$ years). Median number of patients and number of new patients per outpatient clinic session were 40 (range 10 200) and 5 (range 2 12.5), respectively. Mean duration of consultation allocated to a new and an established patients were 12.3 (SD 5.4) and 4.8 (SD 1.8) minutes, respectively.

Responses about the adequacy of consultation time and

Table 2. The response on consultation length

\begin{tabular}{|c|c|}
\hline Variable & Value \\
\hline \multicolumn{2}{|c|}{$\begin{array}{l}\text { Do you have discretion for allocating the consultation } \\
\text { time per patients? }(n=106)\end{array}$} \\
\hline Always & $5(4.7)$ \\
\hline Sometimes & $32(30.2)$ \\
\hline Rarely & $53(50)$ \\
\hline Never & $16(15.1)$ \\
\hline \multicolumn{2}{|c|}{$\begin{array}{l}\text { Does insufficient consultation time affect the quality of } \\
\text { patient care? }(n=96)\end{array}$} \\
\hline No & $1(1.0)$ \\
\hline Yes & $95(99.0)$ \\
\hline \multicolumn{2}{|l|}{$\begin{array}{l}\text { Has insufficient consultation time led to any of the } \\
\text { following? }(n=95)\end{array}$} \\
\hline Unnecessary prescription & $47(49.5)$ \\
\hline Unnecessary test & $68(71.6)$ \\
\hline Unnecessary hospitalization & $44(46.3)$ \\
\hline \multicolumn{2}{|l|}{$\begin{array}{l}\text { What aspects of patient care is negatively affected by } \\
\text { insufficient consultation time? }(n=106)\end{array}$} \\
\hline Treatment decision & $55(51.9)$ \\
\hline Doctor-patient relationship & $72(68.0)$ \\
\hline Patient safety (including error in prescription) & $70(66.0)$ \\
\hline Patient education & $89(84.0)$ \\
\hline Proper documentation & $81(76.4)$ \\
\hline
\end{tabular}

Values are presented as number (\%). related issues are shown in Table 2 and Figure 1. For new and established patients, $78.1 \%$ and $83.1 \%$ of respondents responded that the consultation time is insufficient. On the other hand, only $4.7 \%$ responded that they always have autonomy for the allocation of consultation time for patients. A majority of respondents (61.3\%) replied that they have been restrained from keeping the adequate outpatient clinic volumes by the hospital executive. More than half of respondents responded that they experienced the following: allocation of more than one patient at the same time $(66 \%)$, allocation of less than 5 minutes for a new patient (64.2\%), allocation of more than 10 patients in an hour (73.5\%). Most of the respondents (99.0\%) who responded that the consultation time is inadequate replied that short consultation time leads to low quality of patient care. Sixty six percent of respondents replied that inadequate consultation time affects patient safety including errors in prescription, while $71.6 \%$ replied that it leads to unnecessary tests.

Table 3 shows perceived ideal consultation time for RA and SLE patients. On average, optimal consultation time for new and established RA patients were 34.8 and 17.6 minutes, respectively. That for new and established SLE patients was 38.8 and 21.6 minutes, respectively. Thus, rheumatologists were allocated only $22 \% \sim 35.3 \%$ of optimal consultation time during actual practice.

Table 4, Figure 1 and Figure 2 show responses on actual outpatient practice environment. Only $15.7 \%$ and $18.6 \%$ responded that they have enough time to wash hands and to go to bathroom between patients. Thirty five percent responded that their outpatient clinic never finishes be-

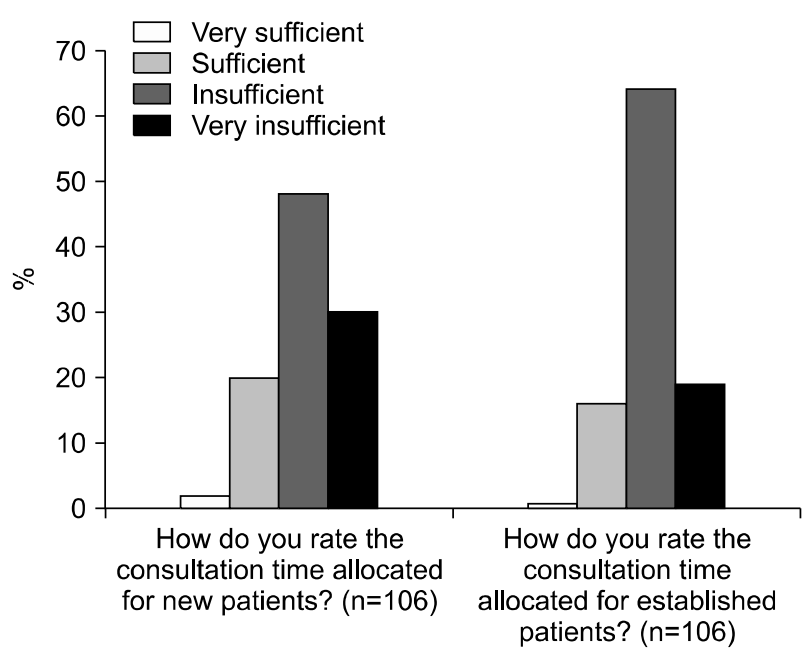

Figure 1. The response on consultation length. 
Table 3. Perceived ideal duration of consultation time (minutes)

\begin{tabular}{|c|c|}
\hline Variable & Value \\
\hline \multicolumn{2}{|l|}{ Rheumatoid arthritis (new patient) } \\
\hline Pre-consultation review & $7.9 \pm 10.4$ \\
\hline History taking & $7.3 \pm 4.6$ \\
\hline Physical examination & $5.1 \pm 3.1$ \\
\hline Treatment planning and prescription & $4.1 \pm 2.9$ \\
\hline Education & $5.9 \pm 3.8$ \\
\hline Documentation & $4.6 \pm 2.8$ \\
\hline \multicolumn{2}{|l|}{ Rheumatoid arthritis (established patient) } \\
\hline Pre-consultation review & $2.9 \pm 2.0$ \\
\hline History taking & $3.2 \pm 2.0$ \\
\hline Physical examination & $3.0 \pm 2.3$ \\
\hline Treatment planning and prescription & $2.6 \pm 1.3$ \\
\hline Education & $3.3 \pm 2.3$ \\
\hline Documentation & $2.6 \pm 2.0$ \\
\hline \multicolumn{2}{|l|}{ Systemic lupus erythematosus (new patient) } \\
\hline Pre-consultation review & $8.1 \pm 5.7$ \\
\hline History taking & $8.3 \pm 5.1$ \\
\hline Physical examination & $5.3 \pm 3.4$ \\
\hline Treatment planning and prescription & $4.4 \pm 2.6$ \\
\hline Education & $7.6 \pm 4.4$ \\
\hline Documentation & $5.1 \pm 4.2$ \\
\hline \multicolumn{2}{|c|}{ Systemic lupus erythematosus (established patient) } \\
\hline Pre-consultation review & $3.6 \pm 2.6$ \\
\hline History taking & $3.8 \pm 2.4$ \\
\hline Physical examination & $3.8 \pm 5.9$ \\
\hline Treatment planning and prescription & $3.2 \pm 2.3$ \\
\hline Education & $4.1 \pm 2.6$ \\
\hline Documentation & $3.1 \pm 2.1$ \\
\hline
\end{tabular}

Values are presented as mean \pm standard deviation.

Table 4. The response on the practice envirornment

\begin{tabular}{lc}
\hline \multicolumn{1}{c}{ Variable } & \multicolumn{1}{c}{ Value } \\
\hline \multicolumn{2}{c}{ Does your hospital administration force you to increase } \\
patient volume? $(\mathrm{n}=102)$ \\
No & $29(28.4)$ \\
Yes & $73(71.6)$ \\
Do you rest between 1 hour of patient care? $(\mathrm{n}=102)$ & $100(98.0)$ \\
No & $2(2.0)$ \\
Yes & \\
Do you have enough time to go to bathroom during patient \\
care? ( $\mathrm{n}=102)$ \\
No & \\
Yes & $19(18.4)$ \\
\hline
\end{tabular}

Values are presented as number (\%).

fore lunch time. About the subjective problems experienced during outpatient clinics, $62.9 \%$ responded that they experience problems in decision making because of exhaustion, while $33.3 \%$ responded that they experienced anxiety. About seven percent reported panic attack



Figure 2. The response on the practice environment.

during practice.

\section{DISCUSSION}

In this study of hospital-based rheumatologists, the mean duration of consultation allocated to a new and an established patient was found to be 12.3 and 4.8 minutes, which corresponded to only $22 \% \sim 35.3 \%$ of perceived optimal consultation time. The majority of respondents reported that they did not have discretion in allocation of consultation time for each patient nor in keeping the adequate outpatient clinic volumes. Insufficient consultation time was found to have negative impact in a vast array of patient care including errors in prescription and overuse of laboratory test.

Although it is generally believed that a sufficient consultation time has a positive influence on patient satisfaction, consultation length often represents how physicians balance contradictory concerns such as productivity and quality of care. Most previous reports on consultation length and its influence on patient care derive from primary care, while scare data exist on specialty care including rheumatology. In addition, it is not known what is the minimum length of consultation that does not compromise patient care. A recent study suggested that 5 minutes may be the limit below which consultations amount to little more than triage and the issue of prescriptions [6]. In that regard, mean consultation time of 4.8 minutes for established patients with rheumatologic 
disease in our survey of rheumatology specialist is of great concern. Consultation length depends on many variables including both patient-related and practitioner-related ones. A study of Swedish general practitioners showed that short consultations were often made by younger male patients about physical problems, while long consultations often were made by older patients about problems with both psychological and physical dimensions [7]. A study of UK general physicians showed that female patients and physicians were significantly more likely to have longer consultation time. Aside from the intrinsic complexity of rheumatologic diseases, these demographic features fit the majority of patients seen by rheumatologists. In our study, rheumatologists responded that adequate consultation time for new and established RA patients were 34.8 and 17.6 minutes, respectively, and 38.8 and 21.6 minutes, for new and established SLE patients. As much time for pre-consultation review, education and documentation was needed as for history taking, physical examination, treatment planning and prescription.

Excessively short consultation leads to numerous problems that negatively affect patient care. For example, in a study in Pakistan involving 996 encounters between children with diarrhoea, general practitioner who spend on average $3 \pm 2$ minutes were far more likely to prescribe antibacterials, antidiarrhoeals and antiamoebics compared to pediatricians who spend $9 \pm 4$ minutes [8]. Another study from India showed that short consultation length was responsible for driving polypharmacy, and poor communication with patients [9]. On the other hand, an association between longer consultation length and reduction in hospitalization for diabetes was reported [1]. In line with these result, a significant proportion of the respondents in our survey replied that they have experienced prescription of unnecessary medication and tests and even unnecessary hospitalization. In addition, insufficient consultation time negatively affects patient safety as well as doctor patient relationship, which all in all leads to huge wastes in healthcare. Short consultation also takes its toll on physicians. Short consultation length related with physical burnout of primary physicians is reported, which may indicate that doctors feel less productive and competent at managing complex multimorbid patients [1]. In a study investigating the amount of physician workload in the outpatient practices of 339 urologists, rheumatologists, and general internists, visits with shorter encounter times were found more labor-intensive than longer ones, such that reduction of a $15 \mathrm{mi}$ - nutes-consultation for an established outpatient to a 10 minutes one led to $26 \%$ increase in workload [10]. It is of no surprise that a significant number of respondents in our survey reported physical symptoms such as anxiety and even panic during outpatient clinic session. All of these negative aspects on physicians undoubtedly affect patient care.

It was of great concern that despite the extremely insufficient consultation length and overbooking of outpatient clinic in hospitals, the majority of respondents replied that they were forced to increase patient volume in the clinic by the hospital executives. This behavior on behalf of hospital executives not only undermines care quality, which should be the priority in the tertiary hospitals, but also is detrimental to health care delivery system of the nation as a whole, which is already distorted to a worrisome extent. Currently, very low compensation for physician cognitive service such as consultation compared to laboratory and imaging is considered one of the culprits perpetuating this problem. For example, compared to consultation fee, laboratory fee such as hemoglobin A1c is very high in Korea (at $50 \%$ of consultation fee compared to $10 \%$ and $17 \%$ in the US and Japan, unpublished data). A study comparing physician wages reflected in the Korean relative value unit system with the Korean hourly minimum wage showed that Korean physician fees are 2.8 3-fold higher than the minimum wage [11]. As a reference, physician consultation fee for US Medicare and Medicaid, which defines a minimum flat physician fee publicly, are on average $22.4 \sim 22.9$ fold. On the other hand, they are on average 9.2 9.8 fold higher than minimum wage in Australia, Germany and France. Low level of compensation for physician consultation leads to distortion of health care service that depend on better compensated services, such as laboratory tests and imaging. Appropriate compensation which guarantees sufficient consultation time, as well as strong policy to control excessive profit-driven management policy of the hospitals would be the only way to solve the current problem.

Our study has limitations. First, despite all the efforts to increase response rates, the overall response rate was less than half of eligible subjects. Second, only rheumatologists working in a hospital setting were included, because of the perceived difference in practice pattern and autonomy in practice. 


\section{CONCLUSION}

Our results showed that rheumatology consultation time is very insufficient compared to what rheumatologists perceive as adequate. A drastic change in health care policy promoting good quality of care is urgently needed.

\section{ACKNOWLEDGMENTS}

This work was supported by the research fund of Rheumatology Research Foundation (RRF-2017-06).

\section{CONFLICT OF INTEREST}

No potential conflict of interest relevant to this article was reported.

\section{AUTHOR CONTRIBUTIONS}

Conception and design of study, acquisition of data, analysis and/or interpretation of data, drafting the manuscript, revising the manuscript critically for important intellectual content: H.A.K. Conception and design of study, acquisition of data, analysis and/or interpretation of data: M.G.K.

\section{SUPPLEMENTARY DATA}

Supplementary data can be found with this article online at http://www.jrd.or.kr and at https://doi.org/10.4078/ jrd.2020.27.1.45.

\section{REFERENCES}

1. Irving G, Neves AL, Dambha-Miller H, Oishi A, Tagashira $\mathrm{H}$, Verho $\mathrm{A}$, et al. International variations in primary care physician consultation time: a systematic review of 67 countries. BMJ Open 2017;7:e017902.

2. Hutton C, Gunn J. Do longer consultations improve the management of psychological problems in general practice? A systematic literature review. BMC Health Serv Res 2007;7:71.

3. Mercer SW, Fitzpatrick B, Guthrie B, Fenwick E, Grieve E, Lawson K, et al. The CARE Plus study - a whole-system intervention to improve quality of life of primary care patients with multimorbidity in areas of high socioeconomic deprivation: exploratory cluster randomised controlled trial and cost-utility analysis. BMC Med 2016;14:88.

4. Wilson AD, Childs S. Effects of interventions aimed at changing the length of primary care physicians' consultation. Cochrane Database Syst Rev 2006; (1):CD003540.

5. Vos T, Flaxman AD, Naghavi M, Lozano R, Michaud C, Ezzati M, et al. Years lived with disability (YLDs) for 1160 sequelae of 289 diseases and injuries 1990-2010: a systematic analysis for the Global Burden of Disease Study 2010. Lancet 2012;380:2163-96.

6. Iacobucci G. GP appointments last less than five minutes for half the world's population. BMJ 2017;359:j5172.

7. Andersson SO, Ferry S, Mattsson B. Factors associated with consultation length and characteristics of short and long consultations. Scand J Prim Health Care 1993;11:61-7.

8. Nizami SQ, Khan IA, Bhutta ZA. Drug prescribing practices of general practitioners and paediatricians for childhood diarrhoea in Karachi, Pakistan. Soc Sci Med 1996;42:1133-9.

9. Chattopadhyay A. An audit of prescribing practices in CGHS dispensaries of Kolkata, India. IOSR J Dent Med Sci 2013;8:32-7.

10. Lasker RD, Marquis MS. The intensity of physicians' work in patient visits--implications for the coding of patient evaluation and management services. N Engl J Med 1999;341: 337-41.

11. Kim HA, Jung SH, Park IY, Kang SH. Hourly wages of physicians within medical fees based on the Korean relative value unit system. Korean J Intern Med. Forthcoming. 